\title{
Ion current distribution on a substrate during nanostructure formation
}

\author{
I Levchenko ${ }^{1}, M_{\text {Korobov }}{ }^{1}, M \operatorname{Romanov}^{1}$ and M Keidar ${ }^{2}$ \\ ${ }^{1}$ National Aerospace University, Kharkov, Ukraine \\ ${ }^{2}$ Department of Aerospace Engineering, The University of Michigan, Ann Arbor, MI 48109, \\ USA \\ E-mail: iglev@ukr.net
}

Received 26 December 2003

Published 26 May 2004

Online at stacks.iop.org/JPhysD/37/1690

DOI: $10.1088 / 0022-3727 / 37 / 12 / 014$

\begin{abstract}
The distribution of an ion current density on a substrate with a discontinuous film consisting of islands of dimension $25-100 \mathrm{~nm}$ was studied numerically. It was found that the ion current distribution is highly non-uniform and islands of the discontinuous film are enclosed with sharply defined zones where the current density is very low. The total area of low current zones reaches $30-50 \%$ of the substrate area, depending on the voltage applied to the substrate. The ratio of island radius-low current zone width depends on the island size and equals approximately 1.0 for large islands and 0.5 for small islands. It is shown that the island distribution function may be controlled by bias voltage variation.
\end{abstract}

\section{Introduction}

Thin films are of great interest for various technical applications [1-4]. Film properties are determined by the film structure, which depends on the sizes and size distribution of the islands forming a film $[5,6]$. Usually a film consists of isolated islands of various sizes during the initial stage of deposition. These islands grow due to particle influx to the island and the substrate surface. The particles that hit upon the substrate will then diffuse on the substrate surface and form a surface flow to the island border [7]. A subsequent growth of isolated islands on a surface causes their contact and coalescence $[8,9]$, and a continuous film is formed.

Ion deposition is one of the prospective methods that allow film application on various substrates. A film structure may be controlled in this process by changing the ion flux parameters, such as the ion current density and ion energy [10]. In plasma immersion technologies the ion flux is extracted from a plasma and accelerated in the sheath formed between the plasma and substrate $[11,12]$. In this case the coating is formed from a high-energetic ion flux, and the film and substrate temperatures, as well as film chemical composition, can be controlled.

One way of studying the film formation process is numerical simulation, which is intensively used now $[13,14]$.
Both analytical and numerical investigations of film formation represent a problem due to the great number of physical effects involved [15-17]. Models that use the concept of a generalized diffusion field can be used for situations where the coverage, i.e. proportion of substrate area covered by the discontinuous film, is much less than 1 . When the coverage (ratio of total island area to substrate area) exceeds 0.1 , the concept of a generalized diffusion field cannot provide the sufficient accuracy due to the interaction between local diffusion fields created by neighbouring islands [5]. In this case one should examine the growth conditions for each particular island for calculating the surface concentration of absorbed atoms and hence the atom flux to each island border. This approach is employed in this work, in which we consider an island ensemble with a coverage of about 0.2. In particular we show that the ion current density over the substrate surface is essentially non-uniform and should be taken into account during modelling of the film formation process.

The rest of the paper is organized as follows. In section 2 a model is presented for numerical simulation of the ion current density on a substrate where an island pattern with mean radius $36 \mathrm{~nm}$ is formed. In section 3 the initial and boundary conditions and in section 4 the numerical method are described. In section 5 the results obtained are described and an interpretation is proposed. 


\section{Model}

The calculation of the ion current density over the substrate surface is based on the island pattern formation model described in detail elsewhere [5]. The island growth rate was calculated on the basis of growth and diffusion equations for the adatom surface concentration:

$$
\begin{gathered}
\frac{\partial V_{\mathrm{i}}}{\partial r_{\mathrm{i}}} \mathrm{d} r_{\mathrm{i}}=J_{\mathrm{a}} \mathrm{d} t \\
\frac{\partial \eta}{\partial t}=D_{\mathrm{a}}\left(\frac{\partial^{2} \eta}{\partial x^{2}}+\frac{\partial^{2} \eta}{\partial y^{2}}\right)+j_{\mathrm{ae}}-j_{\mathrm{ve}}
\end{gathered}
$$

where $V_{\mathrm{i}}$ is the island volume; $r_{\mathrm{i}}$ is the island radius; $J_{\mathrm{a}}$ is the total volumetrical flow to the island; $t$ is the time; $j_{\mathrm{ae}}$ is the external flow to the substrate surface; $j_{\mathrm{ve}}$ is the evaporation flow from the substrate surface; $x, y$ are the coordinates; and $\eta\left(x_{0}, y_{0}, t\right)$ is the surface adatom concentration. The details of calculating the flows to the island surface and other values can be found in [5].

If the film being formed is an insulator, the growing islands will bear a positive electrical charge that is created by ions deposited on the island surface. A summarized electrical field, created by a negatively charged substrate and an ensemble of positively charged islands, will affect the motion of ions being deposited to the substrate. Some ions may be deflected from the straight trajectory, dependent on the ion position above the surface, and the uniform deposition may be disturbed.

The ion motion in a summarized electrical field was calculated from the motion equations, written in the form

$$
\begin{gathered}
\vec{r}(t)=\vec{r}_{0}+\int_{t_{0}}^{t} \vec{v}_{\mathrm{i}}(\tau) \mathrm{d} \tau, \\
\vec{v}(t)=\vec{v}_{0}+\int_{r_{0}}^{0} \frac{e \vec{E}}{m_{\mathrm{i}}}(r) \mathrm{d} r,
\end{gathered}
$$

where $\vec{r}(t)$ is the ion position vector; $\vec{r}_{0}$ is the initial position vector; $\vec{v}_{\mathrm{i}}(t)$ is the ion velocity; $t_{0}$ is the initial time of ion movement; $\vec{v}_{0}$ is the initial ion velocity; $e$ is the electron charge, and $m_{\mathrm{i}}$ is the ion mass. Equations (3) and (4) allow explicit integration of the ion trajectories that were used for determination of the ion current density on the substrate surface. The summarized electrical field applied to the ion that is located at point $\vec{r}(t)$ was calculated by integrating the surface charge of all islands on the substrate:

$$
\vec{E}(\vec{r})=\sum_{0}^{n_{\mathrm{i}}} \int_{S_{\mathrm{i}}} \frac{\rho_{\mathrm{i}} \mathrm{d} S_{\mathrm{i}}}{4 \pi \varepsilon_{0} r^{3}} \vec{r}+\frac{U_{\mathrm{S}}}{l_{\mathrm{d}}},
$$

where $\vec{E}(\vec{r})$ is the summarized electrical field vector; $n_{\mathrm{i}}$ is the number on islands on the substrate; $\rho_{\mathrm{i}}$ is the surface density of electrical charge on the island surface; $\vec{r}$ is the radius vector of the point where the electrical field is being calculated (the distance from island to ion); $\varepsilon_{0}$ is the electric constant; $S_{\mathrm{i}}$ is the island surface; $l_{\mathrm{d}}$ is the Debye layer between the substrate and plasma; $U_{\mathrm{S}}$ is the voltage applied to the substrate, and hence $U_{\mathrm{S}} / l_{\mathrm{d}}$ is the electrical field created by the bias voltage applied to the substrate. During this summation, the image electrical charges induced in the conductive substrate by the charged islands were also taken into account. A low ion current density was considered, and so the space charge of the ion current was neglected, and the electrical field produced by the ion flux was not taken into consideration.

In this calculation the islands were assumed to have a disc geometry with a flat upper surface; the disc height was assumed to be less than the radius. The surface density of the electrical charge on the island surface, $\rho_{\mathrm{i}}$, was calculated under the assumption that the island surface is charged by the ion flux accelerated with voltage $U_{\mathrm{S}}$ applied to the substrate. The effect of the secondary ion-electron emission was neglected in this model since the electron emission yield does not exceed 0.1 for ion energies not exceeding $1000 \mathrm{~V}$ [18]. So each island acquires the potential $U_{\mathrm{S}}$, and we have the following equation for $\rho_{\mathrm{i}}$ :

$$
U_{\mathrm{S}}=\int_{0}^{\infty} \int_{S_{\mathrm{i}}} \frac{\rho_{\mathrm{i}} \mathrm{d} S \mathrm{~d} z}{4 \pi \varepsilon_{0} z^{2}},
$$

where $z$ is the distance to the island, measured along a perpendicular to the substrate surface. The system of equations (3)-(6) describes the summarized electrical field and ion trajectories over the substrate surface for the island pattern calculated with the help of equations (1) and (2) and may be used for numerical calculation of the ion current distribution on the substrate surface.

\section{Initial and boundary conditions}

The system (3)-(6) should be complemented with initial and boundary conditions. In these calculations we have assumed that the electrical field is applied across the Debye layer between the substrate and plasma:

$$
l_{\mathrm{d}}=\sqrt{\frac{\varepsilon_{0} T_{\mathrm{e}}}{n_{\mathrm{e}} e}},
$$

where $T_{\mathrm{e}}$ is the electron energy $(\mathrm{eV}) ; n_{\mathrm{e}}$ is the plasma density outside the Debye layer $\left(\mathrm{m}^{-3}\right)$; thus, the starting plane for the present simulations was assumed at the sheath edge, $l_{\mathrm{d}}$, calculated from equation (7). For the calculations we used an example with a typical plasma density of about $10^{18} \mathrm{~m}^{-3}$ and electron temperature of about $T_{\mathrm{e}}=3 \mathrm{eV}$, assuming that the plasma is produced by the vacuum arc source [19]. These conditions provide a Debye layer of about $10^{-5} \mathrm{~m}$, which is much larger that the mean island radius.

The initial ion position in the $x-y$ plane was determined as random numbers in the range of $0<x<\lambda_{\mathrm{s}}, 0<y<\lambda_{\mathrm{s}}$, where $\lambda_{s}$ is the length of substrate area used in calculations. The initial condition for ion motion equations consists of specifying the initial velocity for the ion that enters the Debye layer. Assuming that ions enter the sheath with Bohm velocity, we will have $v_{0}=\sqrt{T_{\mathrm{e}} \cdot e / m_{\mathrm{i}}}$ for an electron energy of $T_{\mathrm{e}}=3 \mathrm{eV}[20]$.

\section{Numerical simulation}

The calculations were made on a surface area covered with a mesh of $500 \times 500$ cells with a cell size of $10 \lambda$, where the lattice constant, $\lambda$, was assumed to be $5 \times 10^{-10} \mathrm{~m}$. The calculation sequence was as follows: first, the island pattern was determined from equations (1) and (2) according to the 
methodology described in [5]; second, the surface charge was calculated for every island from the ensemble using equation (6); third, the ion trajectory was calculated using equations (3)-(5), starting from height $z_{0}=l_{\mathrm{d}}$ and random coordinates $x$ and $y$. The ion motion in the summarized electrical field was traced until ion impact with the substrate surface. To obtain the current distribution on the surface, we traced up to 200000 ions. The coordinate of ion collision with the substrate surface was accumulated in a current density matrix. On completion of the calculations the density matrix was imaged, the areas with higher current density coloured with lighter hues and vice versa.

The ion current distribution was calculated for a substrate bias voltage $\left(U_{\mathrm{S}}\right)$ of about $200 \mathrm{~V}$. Besides, in order to study the dependence of the ion current distribution on the island charge, we made additional calculations under the assumption that the short pulses of higher voltage, $U_{\mathrm{P}}$, are applied periodically to the substrate. We assumed that the duration of the pulse voltage, $U_{\mathrm{P}}$, is short in comparison with the interval between pulses (small duty cycle), and so we considered the ion deposition only between pulses. Hence, charge accumulation on the island is being made mainly during high voltage pulses, and the film deposition occurs under the surface charge conditions that were created during an increased voltage pulse. These pulses provide increased electrical charge on the island surface, which determines the electrical field according to equations (3) and (4) (the voltage $U_{\mathrm{P}}$ is used in this case instead of $U_{\mathrm{S}}$ in equation (4). The calculations were performed for pulse voltages, $U_{\mathrm{P}}$, of $300,400,500$ and $1000 \mathrm{~V}$.

\section{Results and discussion}

The ion current density on the substrate surface was calculated for the nanometre-size island pattern shown in figure 1 . The distribution function of islands is close to Gaussian with a mean island radius of about $75 \lambda(36 \mathrm{~nm})$, and the surface coverage is about 0.2 . The details on the model and the island growth conditions are described in [5].

Typical ion trajectories calculated by integrating the ion motion equations are shown in figure 2. From this figure one can see that there are three types of ion trajectories. When an

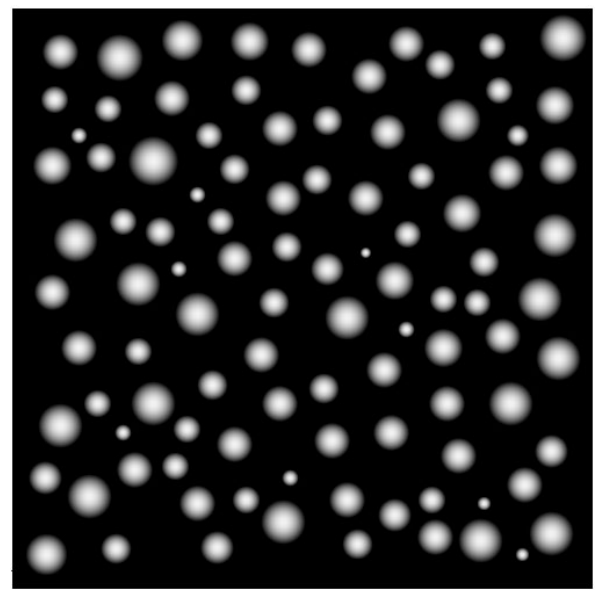

Figure 1. The islands pattern used for calculation of the ion current density on the substrate surface. ion starts to move to the substrate over an island that is not closely surrounded by other islands, it deflects from the island centre and moves away from the island border to a distance comparable with the island diameter ( 1 in figure 2$)$. When an ion starts to move over a point located between the islands (over the uncovered surface), it does not deflect significantly from a vertical pass ( 2 in figure 2 ). When an ion starts to move between closely located islands ( 3 in figure 2), it changes significantly the direction of movement and sometimes makes a U-turn.

The topography of the electrical field calculated from equation (3) is shown in figure 3 . It can be seen that the islands are surrounded by an electrical field that is high along the island border but becomes much lower between islands.

The topography of the ion current density on the substrate surface is shown in figure 4 . The particular pattern of current density depends on the $U_{\mathrm{P}} / U_{\mathrm{S}}$ ratio, but some peculiarities are generic and may be observed in this three-dimensional image. From this image one can see that the islands (shown as flat bulges) are enclosed with zones where the ion current density is

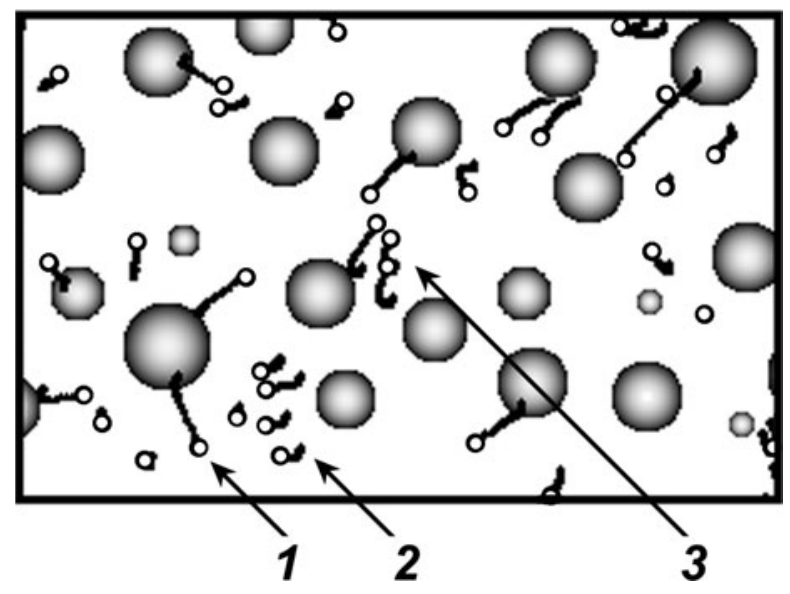

Figure 2. Typical ion trajectories (black lines-trajectories, small circles - points of ion collision with substrate). 1, strong deflection; 2 , weak deflection; 3 , considerable change in direction of movement.

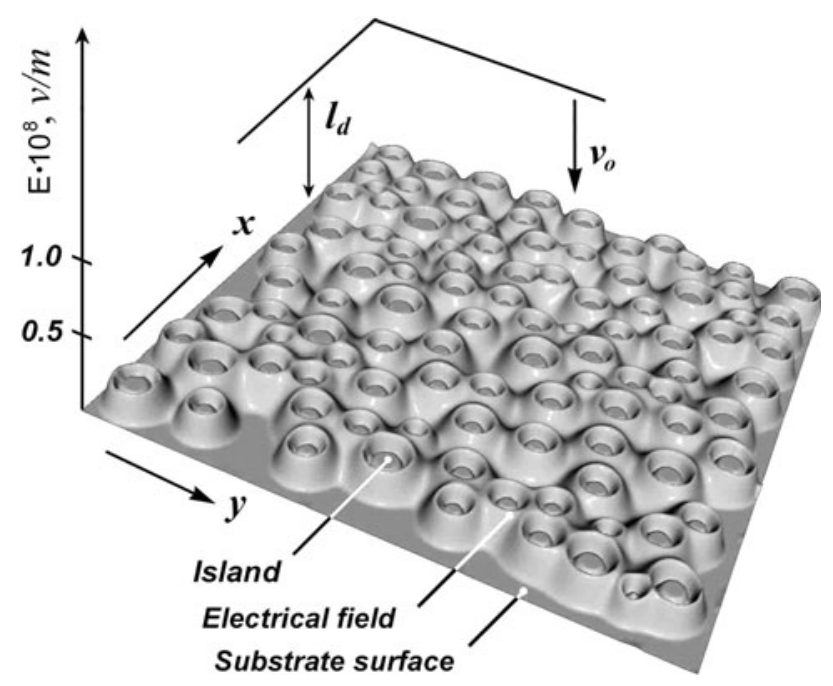

Figure 3. Three-dimensional image of electrical field in island system for bias voltage $U_{\mathrm{S}}=300 \mathrm{~V}$. The islands are shown conditionally as flat bulges. 
very low (tends to zero). These zones are circled with elevated ridges, and the rest of the surface of the substrate is covered with a relative high current density, where the electrical field is low.

In order to study the ion current density distribution over the entire substrate surface, we made a simulation for various $k_{\mathrm{u}}=U_{\mathrm{P}} / U_{\mathrm{S}}$ ratios, in the range $k_{\mathrm{u}}=1-5$. The patterns of ion current density corresponding to different $k_{\mathrm{u}}$ are shown in figures $5(a)-(c)$. From these figures one can see that the mean width of the low current density zones increases with $k_{\mathrm{u}}$. This is the direct result of the presence of electrical charge on the island surface accumulated during the high voltage pulse. When the voltage ratio is about 1 (no high voltage pulse), the low current density zones cover an area that is equal to the total area of islands (a zone width is comparable with the island radius, figure $5(a)$ ), and the influence of these zones on the island growth should be appreciable. When the voltage ratio, $k_{\mathrm{u}}$, increases, the low current zones expand and a high current ridge is formed around the low current zones (figure 5(b), also visible in figure 4 ). With the voltage ratio, $k_{\mathrm{u}}$, reaching 2 , the low current zones expand to the entire substrate, the ridges close up and the ion current is concentrated mainly in thin fascias located equidistantly between the islands. So $k_{\mathrm{u}}=2$ represents the limit for useful application of high voltage pulses. A further increase in the voltage ratio, $k_{\mathrm{u}}$, causes the

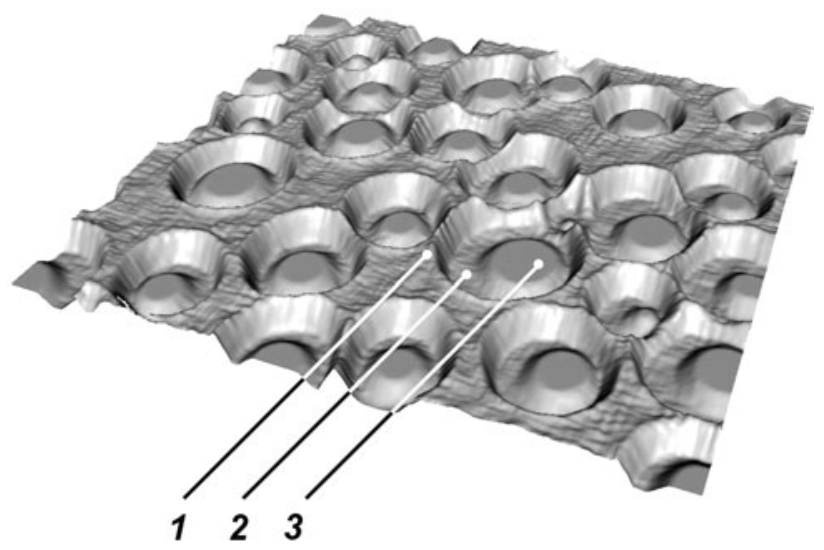

Figure 4. Three-dimensional image of relative ion density distribution in island system. 1, zone of high current density; 2 , zone of low current density; 3 , island. formation of thinner ridges, and finally, the ion current to the substrate surface stops when the voltage ratio, $k_{\mathrm{u}}$, reaches 5 $\left(U_{\mathrm{P}}=1000 \mathrm{~V}\right.$ for $\left.U_{\mathrm{S}}=200 \mathrm{~V}\right)$. This means that deposition is impossible in this case up to the moment when the excessive electrical charge leaks from the island surface and the ionretarding field is decreased. Even at $k_{\mathrm{u}}=1$ (figure 5(a)) not all ions reach the substrate, but some ones are pushed out of the Debye layer.

It should be noted that we consider the deposition between pulses of high voltage, $U_{\mathrm{P}}$, when the electrical charge is kept on the island surface. During the deposition at voltage $U_{\mathrm{S}}$, the increased charge created by pulse voltage $U_{\mathrm{P}}$ will leak gradually from the island surface due to some conductivity of the island material (very small since we consider the deposition of a non-conducting film). When the electrical charge of the island decreases, a new pulse should be applied to restore the island charge state; so the steady charge on the island is maintained. The process of charge leak from the islands surface was not considered in this work, but we assumed that the duration of the pulsed voltage, $U_{\mathrm{S}}$, is much smaller than the period between pulses (on-off ratio is much less than 1), and so a steady state of the electrical charge on the islands can be considered. When no pulses of increased voltage are used $\left(k_{\mathrm{u}}=1\right.$, figure $\left.5(a)\right)$, the real steady state conditions are applied.

The dependence of the width of the low current zone on the island radius is shown in figure 6 . This dependence was plotted by examining the calculated ion current density distribution on the surface. The graph shows that the width of the low current zone, $r_{\mathrm{z}}$, increases with the island radius. For small islands $\left(r_{\mathrm{i}}=0.05 \mu \mathrm{m}\right)$ the width of the low current zone reaches $0.04 \mu \mathrm{m}$ for the case $k_{\mathrm{u}}=1$ and $0.07 \mu \mathrm{m}$ for the case $k_{\mathrm{u}}=2$. For large islands $\left(r_{\mathrm{i}}=0.1 \mu \mathrm{m}\right)$, the width of the low current zone reaches $0.1 \mu \mathrm{m}$ for the case $k_{\mathrm{u}}=1$ and $0.16 \mu \mathrm{m}$ for the case $k_{\mathrm{u}}=2$. It is seen that the width of the low current zone is always comparable with the island radius and even exceeds it for higher voltage ratios, $k_{\mathrm{u}}$.

To illustrate the relative change of the ion current density on the various substrate areas, we plotted the dependence of the relative current, $\bar{j}(\delta)=j_{\delta} / j_{\Sigma}$, on distance, $\delta$ (figure 7), where $j_{\delta}$ is the ion current density on a substrate area remote from island borders by a distance of $\delta$ or more (grey area in the scheme shown in lower left corner of figure 7), and
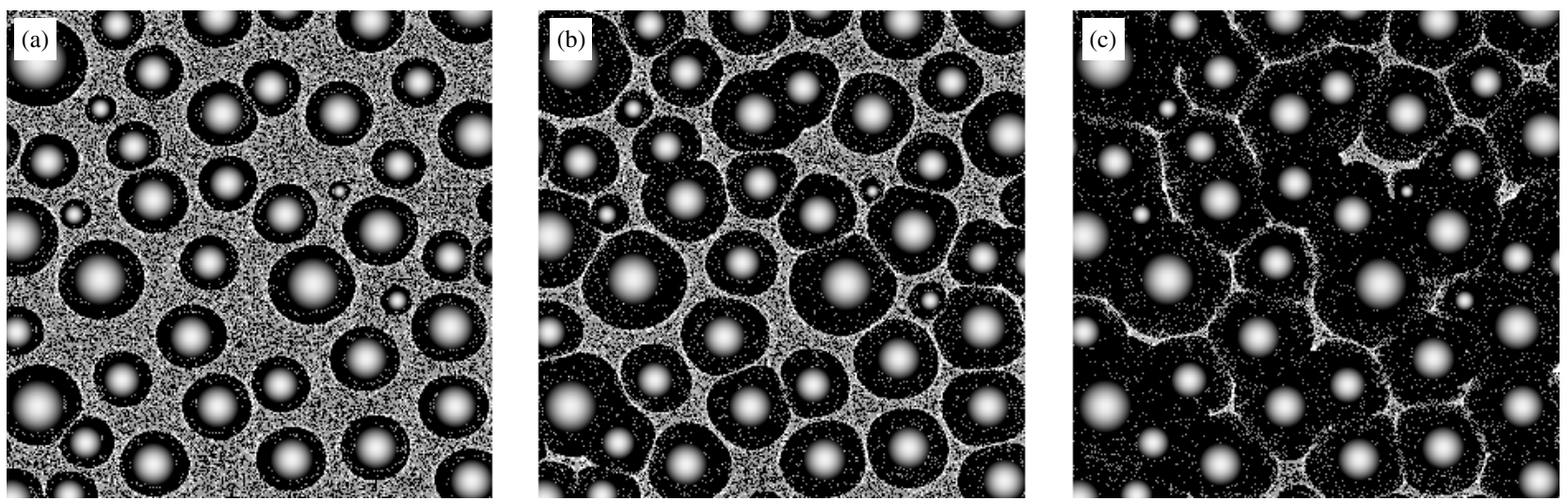

Figure 5. Ion density distribution in island system. Islands are shown as round bulges, the ion current density is rendered in grey colours. (a) $k_{\mathrm{u}}=1.0 ;$ (b) $k_{\mathrm{u}}=1.5 ;$ (c) $k_{\mathrm{u}}=2.0 . U_{\mathrm{S}}=200 \mathrm{~V}$. 


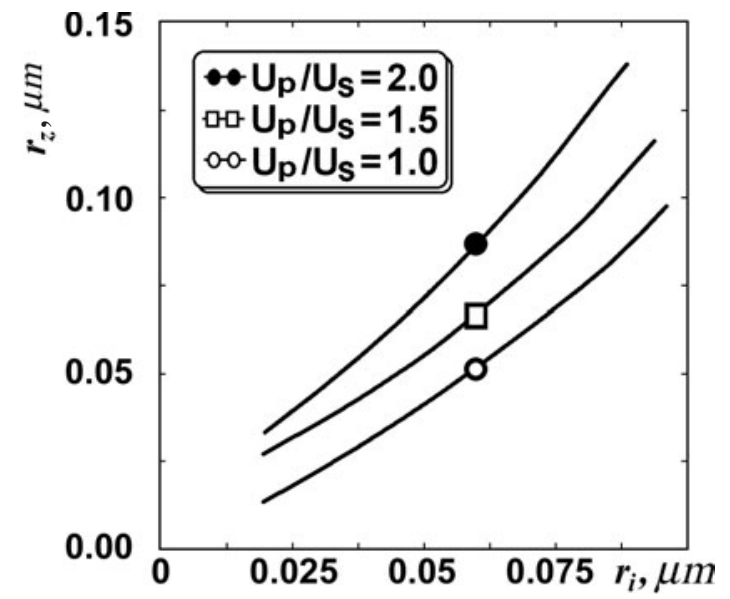

Figure 6. Dependence of width of low current zone, $r_{\mathrm{z}}$, on island radius, $r_{\mathrm{i}}$, with voltage ratio $k_{\mathrm{u}}$ as a parameter.

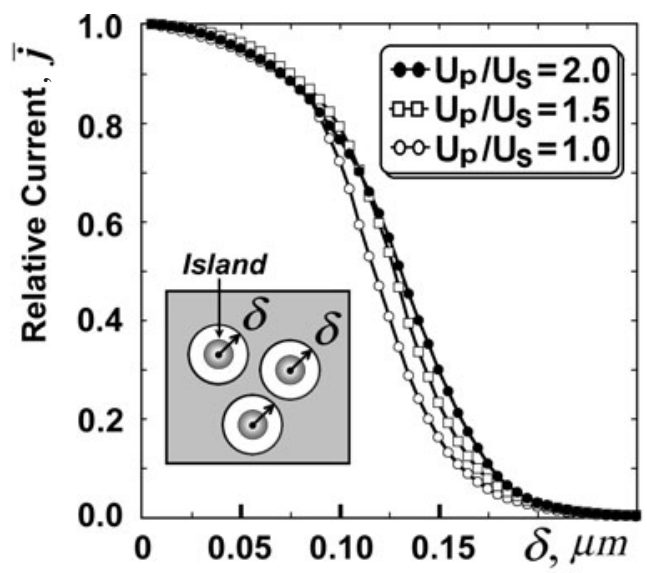

Figure 7. Dependence of total current on distance, $\delta$.

$j_{\Sigma}$ is the total current on the entire substrate surface. This graph shows that the relative current decreases with distance $\delta$. When $\delta$ increases (this means that the surface areas more and more remote from islands are being considered), the relative current decreases weakly for $\delta<0.1 \mu \mathrm{m}$ and sharply when $\delta$ exceeds $0.1 \mu \mathrm{m}$. This value represents, in a certain sense, the mean-integral size of low current zones that enclose the islands. According to this graph one can conclude that the mean-integral size of low current zone varies from $0.7 \mu \mathrm{m}$ for $k_{\mathrm{u}}=1$ to $0.1 \mu \mathrm{m}$ for $k_{\mathrm{u}}=2$. These results correlate with the data shown in figure 6 .

The above studies show that the low current zones formed around the islands during the ion deposition are comparable with the island radius and should be taken into account when the film growth is calculated. When the ratio $k_{\mathrm{u}}$ is small (up to 1.5 , figures $5(a)$ and $(b)$ ), the effect of a uniform ion current distribution provides conditions for equalization of the island distribution function. Indeed, the wide low current zones around the large islands decrease significantly the flow of adsorbed atoms to the island border and hinders the island growth. Conversely, the narrow low current zone around the small islands provides a higher adatom flux to the island border and promotes an increased rate of growth for the small islands. As a result, the smallest islands grow with an increased rate, and the largest islands do not grow. The distribution function becomes more uniform, consisting of islands of some medium size. The maximum island size in these conditions will be restricted; this provides the formation of a dense fine-grained film. It has been shown in recent experiments that the use of increased ion energy provides a smaller electrical resistance to deposited silver films [21]. The surface roughness of gold films applied by vacuum arc deposition, i.e. with increased ion energy, also was decreased as compared with the evaporated films [22].

This result may be interpreted as evidence of finer distribution function formation at higher ion energies. In fact, mainly the number of contact points between islands determines the resistance of a discontinuous film. It is evident that a discontinuous film consisting of a large number of small islands will provide a smaller resistance than a film consisting of big ill-contacting islands. Besides, small islands form more tight contact points with each other since the surface activation energy decreases with decreasing radius [23]. When the ratio $k_{\mathrm{u}}$ exceeds 1.5 , the low current zones close up and small islands become immersed into the low current zones formed around the big islands (see figure $5(c)$ ). As a result, the surface flux to the borders of the small islands will be essentially suppressed, and the islands will dissolve. The island distribution function in this case will shift to larger sizes, and the film will be coarse-grained after coalescence.

\section{Conclusions}

In this paper the ion current distribution on a substrate surface during ion deposition has been studied. We have found that the ion current is distributed non-uniformly and low current zones are formed around islands of discontinuous film. It may be concluded that the width of the low current zones around the islands is comparable with the island radius and should influence the process of island growth. The use of high voltage pulses during the deposition may be an effective tool for the island distribution function control.

\section{References}

[1] Singh S K, Lees M R, Singh R K and Palmer S B 2002 J. Phys. D: Appl. Phys. 35 2243-6

[2] Presting H, Konle J, Kibbel H and Banhart F 2002 Appl. Surf. Sci. $188249-58$

[3] Freund H-J 2002 Surf. Sci. 500 271-99

[4] Seki H, Takada M, Tanabe T, Wadayama T and Hatta A 2002 Surf. Sci. 506 23-32

[5] Levchenko I and Baranov O 2003 Vacuum 72 205-10

[6] Fanfoni M, Tomellini M and Volpe M 2001 Appl. Phys. Lett. 78 3424-26

[7] Rosei F and Rosei R 2002 Surf. Sci. 500 395-413

[8] Lin C, Xu Y H, Naramoto H, Wei P, Kitazawa S and Narumi K 2002 J. Phys. D: Appl. Phys. 35 1864-6

[9] Schmitt F, Osipov A V and Hess P 2002 Appl. Surf. Sci. 188 103-9

[10] Levchenko I and Keidar M 2002 Vacuum 66 77-85

[11] Levchenko I, Romanov M and Keidar M 2003 J. Appl. Phys. 94 1408-13

[12] Levchenko I, Romanov M, Baranov O and Keidar M 2003 Vacuum 72 335-44 
[13] Zhang Q Y and Chu P K 2002 Surf. Coat. Technol. 158-159 247-52

[14] Thurstans R E and Oxley D P 2002 J. Phys. D: Appl. Phys. 35 802-9

[15] Girard P, Titkov A N, Ramonda M, Evtikhiev V P and Ulin V P 2002 Appl. Surf. Sci. 201 1-8

[16] Goto M, Kasahara A, Tosa M and Yoshihara K 2003 Surf. Coat. Technol. 168 98-104

[17] Matthews J W 1975 Epitaxial Growth (New York: Academic)

[18] Raizer Yu P 1991 Gas Discharge Physics (New York: Springer)
[19] Boxman R L, Martin P and Sanders D (ed) 1995 Handbook of Vacuum Arc Science and Technology (Park Ridge, NJ: Noyes)

[20] Kutzner J and Miller H C 1992 J. Phys. D: Appl. Phys. 25 686-93

[21] Byon E, Oates T W H and Anders A 2003 Appl. Phys. Lett. 82 1634-36

[22] Bendavid A, Martin P J and Wieczorek L 1999 Thin Solid Films 354169

[23] Moelwyn-Hughes E A 1961 Physical Chemistry (London: Pergamon) 